\title{
EchoGéo
}

$13 \mid 2010$

Afrique, 50 ans d'indépendance : État et territoires

\section{L'Afrique du Sud}

Compte rendu de lecture

Jean-Louis Tissier

\section{CpenEdition}

Journals

Édition électronique

URL : https://journals.openedition.org/echogeo/11997

DOI : 10.4000/echogeo. 11997

ISSN : 1963-1197

Éditeur

Pôle de recherche pour l'organisation et la diffusion de l'information géographique (CNRS UMR 8586)

Référence électronique

Jean-Louis Tissier, «L'Afrique du Sud », EchoGéo [En ligne], 13 | 2010, mis en ligne le 20 septembre 2010, consulté le 03 août 2021. URL : http://journals.openedition.org/echogeo/11997 ; DOl : https:// doi.org/10.4000/echogeo.11997

Ce document a été généré automatiquement le 3 août 2021.

EchoGéo est mis à disposition selon les termes de la licence Creative Commons Attribution - Pas d'Utilisation Commerciale - Pas de Modification 4.0 International (CC BY-NC-ND) 


\title{
L'Afrique du Sud
}

\author{
Compte rendu de lecture
}

\author{
Jean-Louis Tissier
}

Philippe Gervais-Lambony confronte sa connaissance de l'Afrique du Sud aux idées reçues qui se sont construites et diffusées depuis deux décennies sur ce pays qui a vécu une évolution très singulière. Le parti choisi par cette collection n'est pas sans risque puis que les titres des chapitres affichent des assertions suffisamment simples et fortes pour conforter les idées d'un lecteur naïf ou pressé. Ph. Gervais-Lambony retient une vingtaine d'idées reçues, les inscrit entre guillemets, typographie de précaution figurant des sortes de pincettes expérimentales. Exemple : " Nelson Mandela est un héros... ». La figure fondatrice de la nouvelle Afrique du Sud est soumise à l'examen critique qui, derrière l'icône consensuelle, revient sur un itinéraire politique long fait de convictions réaffirmées, d'épreuves marquantes, de maîtrise stratégique et tactique qui sont la fabrique d'un leader : le « héros » est devenu un des éléments fondateurs de la nouvelle nation sud africaine.

2 Cette nouvelle nation sud africaine est bien le sujet de ce court et dense ouvrage. Ph. Gervais-Lambony est un géographe ouvert aux sciences humaines et politiques, aux expressions artistiques contemporaines ce qui lui permet de faire une présentation actualisée de la nouveauté et de la vitalité sud africaines. En ouverture il explique le choix de cette nation post apartheid d'assumer cette appellation lourde «Afrique du Sud » plutôt que d'adopter, comme souvent en Afrique, un nom faisant référence à une identité politique et culturelle précoloniale.

L'ouvrage comprend 4 parties (chacune avec sa part d'idées reçues) :

- La première partie rappelle aux candidats au voyage que les dépliants touristiques sont parfois trompeurs, le veld est un motif paysager à reconnaître dans ses nuances, le climat de ces plateaux austraux peut être frais, l'anglais est utile mais doit composer avec d'autres langues de base. Cette mise au point est précise, elle rappelle les ressources et les contraintes de ce vaste pays austral. Elle souligne que la question des langues avait une 
dimension politique forte dans le régime de l'apartheid et qu'elle la conserve sous d'autres modalités dans le nouveau.

- Dans la seconde partie Une histoire violente qui se termine bien? Ph. Gervais-Lambony se fait historien et politiste. Il reprend les moments d'une histoire coloniale originale car disputée entre les Européens eux mêmes, dont la lutte, ouverte ou non, construit une société ségréguée de fait puis de « droit » en vue de l'exploitation systématique d'un territoire « dégagé » de ses indigènes et doté d'un potentiel unique en Afrique. La fin de l'apartheid est moins un miracle, idée reçue, qu'une mutation politique en partie négociée, dans un contexte international transformé par la disparition de l'Union soviétique. De même que l'ANC n'est pas l'unique acteur politique, idée reçue, d'une vie politique et syndicale animée et diversifiée depuis des décennies. Cette ANC par ailleurs est un parti parcouru par des courants divers que chaque échéance démocratique réactive.

- La troisième partie Société et culture : les temps du changement fait le point sur les transformations post apartheid : quinze ans après, de fortes inégalités sociales subsistent largement corrélées aux origines raciales : revenus, formation, taux de chômage, santé, les politiques menées n'ont pas encore réduit les écarts entre Blancs et non Blancs, les inerties sociales sont plus marquées que dans le domaine politique. Les changements sont amorcés plus qu'accomplis, le diagnostic de 2009 reste circonspect, les faits à retenir par delà les idées reçues sont des tensions sociales, une persistance du sentiment communautaire, le fléau de la violence, l'épidémie du sida. Ces faits sont analysés, contextualisés par Ph. Gervais-Lambony sans complaisance et sans dépit. La création culturelle atteste de la vitalité de la société sud-africaine, au côté des grands écrivains qui ont critiqué et combattu dans leurs œuvres l'apartheid, est apparue une génération d'artistes auteurs qui disent les sensibilités et les réalités contemporaines de l'Afrique du Sud.

- La dernière partie, la plus courte, évalue le poids et la place de l'économie sud africaine en Afrique et dans le monde. L'ancienne Afrique du Sud dominait largement du point de vue économique les autres économies du continent par la valorisation de son potentiel minier et agricole et pas son organisation capitaliste, par exemple les grandes sociétés minières, notamment l'Anglo American, avaient déjà étendues leurs intérêts hors d'Afrique. Là aussi l'héritage était lourd mais il constituait une base solide pour le nouvel état. Celui ci par le biais des sociétés qu'il contrôle directement ou indirectement a élargi ces investissements en Afrique, australe ou non, dans les domaines miniers, énergétique et des services.

4 L'Afrique du Sud dispose de la première économie africaine. Les acteurs économiques sud africains sont largement acquis aux thèses néo libérales, ils ont privilégié la croissance macro économique et limité la redistribution de ses fruits. L'économie sud africaine engagée dans les échanges mondiaux a été frappée par la crise depuis 2008 : baisse du PIB, déséquilibre de la balance commerciale, ce qui s'est traduit par des licenciements dans le secteur minier. Ce constat conduit P. Gervais-Lambony à réévaluer le caractère émergent de cette économie qui conserve des caractères d'une économie en développement dépendante des exportations de produits bruts en valeur et en volume. La récession présente rappelle la fragilité de l'émergence sud africaine. Cette vulnérabilité relative attise les tensions sociales et politiques, et les nouveaux cadres enrichis qui ont profité dans un premier moment des politiques de discrimination positive ne sont pas à l'abri de ces retournements conjoncturels. Certes la puissance demeure, idée reçue, mais le contexte de la mondialisation a engendré des incertitudes. 
5 La conclusion de l'ouvrage est construite sur le constat d'une ambivalence : le chemin parcouru en deux décennies a été considérables en termes de principe, de droits mais il reste du point de vue social, dans tous ses modalités, beaucoup à faire... La pesée réalisée par Ph. Gervais-Lambony est prudente, évitant les écueils de l'irénisme et du catastrophisme.

6 La mise au point courte est un genre difficile. La collection doyenne, du même format "Que-sais-je? » comprend en géographie et en histoire quelques réussites qui sont devenus des classiques. Seul un grand savoir allié à un savoir faire permet d'accomplir la figure imposée.

7 On soulignera dans le registre de l'efficacité concise la fonction des encadrés permettant de nuancer, de préciser certains points : ainsi sur les Zoulous dans la guerre urbaine, le découpage territorial post apartheid, sur la diffusion des armes à feu, sur la musique. Les références artistiques littéraires picturales apportent la «touche » de l'auteur. Pour les lecteurs que ces 128 pages ont laissés sur leur faim d'approfondissement ils pourront aller plus loin grâce à une bibliographie qui est plurilingue et pluri disciplinaire. Ph. Gervais-Lambony y a l'élégance de ne pas renvoyer à ses précédents ouvrages, en revanche il oriente le lecteur vers les études urbaines. La place des chercheurs français y est notable : pourquoi l'Afrique du Sud post apartheid a-t-elle attiré dans les année 90 cette cohorte curieuse et intrépide de jeunes doctorant(e)s ? L'événement politique, territorial social offrait sans doute un champ de recherche pour une géographie décloisonnée où le politique le social et le culturel allaient ensemble...

8 Le son tonitruant des vuvuzelas n'a pas encore fait tomber les barrières socio-spatiales mais il témoigne de l'énergie de cette société sud africaine.

\section{BIBLIOGRAPHIE}

Gervais-Lambony Ph., 2009. L'Afrique du Sud. Editions Le cavalier bleu, Collection idées reçues.

\section{AUTEUR}

\section{JEAN-LOUIS TISSIER}

Jean-Louis Tissier est Professeur à l'université Paris 1. jean-louis.tissier@univ-paris1.fr 